
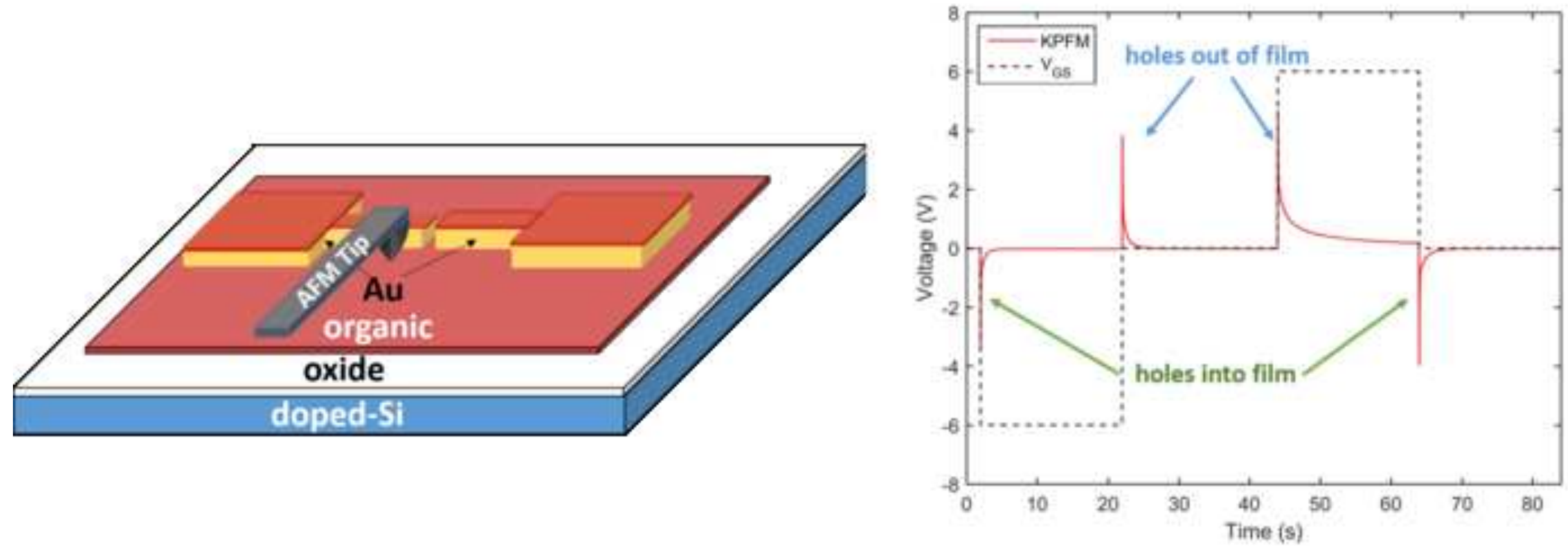


\title{
Time-resolved Kelvin probe microscopy to study population and depopulation of traps in electron or hole majority organic semiconductors
}

\author{
Jason P. Moscatello ${ }^{\mathrm{a}}$, Chloe V. Castaneda ${ }^{\mathrm{a}}$, Alyina Zaidia ${ }^{\mathrm{a}}$, Minxuan $\mathrm{Cao}^{\mathrm{a}}$, \\ Ozlem Usluer $^{\mathrm{b}}$, Alejandro L. Briseno ${ }^{\mathrm{b}}$, Katherine E. Aidala ${ }^{\text {ta } \mathrm{a}}$ \\ ${ }^{a}$ Department of Physics, Mount Holyoke College, 50 College Street, South Hadley, MA, \\ USA \\ ${ }^{b}$ Department of Polymer Science and Engineering, Conte Research Center, University of \\ Massachusetts, 120 Governors Drive, Amherst, MA 01003
}

\begin{abstract}
We present a time-resolved Kelvin Probe Force Microscopy (KPFM) technique that can record carrier motion on the scale of milliseconds, appropriate for polycrystalline materials like organic semiconductors. The organic semiconductors are studied in a transistor geometry to which we apply a step voltage to the back-gate. We record the change in potential at a specific location and observe the times associated with filling and emptying majority carrier traps, observed in hole majority carrier poly(3-hexylthiophene-2,5-diyl) (P3HT) and a perylene diimide electron majority carrier, PDI-CN2. We see signs of bias stress with repeated measurements in $\mathrm{P} 3 \mathrm{HT}$.
\end{abstract}

Keywords: scanning probe microscopy, organic semiconductors, organic photovoltaics, charge motion, P3HT, PDI-CN2

\section{Introduction}

Non-traditional semiconductors like organic materials[1, 2], arrays of colloidal quantum $\operatorname{dots}[3,4]$, metal oxides[5, 6], metal dichalcogenides[7], and perovskites $[8,9]$ are now employed in flexible electronics and next-generation photovoltaics; however, they suffer from low mobilities compared to traditional

Corresponding author. E-mail address: kaidala@mtholyoke.edu (K. Aidala) 
crystalline inorganic materials, and their transport properties are heavily influenced by trap states [2, 3, 4, 5, 6, 7, 8, 9. Substantial work goes into characterizing these trap states for individual materials with techniques that range from illumination-free transient current [10] to (thermal) admittance spectroscopy [1],

Fourier transform photocurrent spectroscopy [12], deep level transient spectroscopy[13], and capacitance-voltage or capacitance-frequency 14 measurements, for example. Depending on the characterization method and the model that is used to interpret the results, these techniques do not always agree, nor are they all sensitive to the same trap states[13]. Kelvin Probe Force Microscopy (KPFM) is typically used to create spatial maps of surface potential, to determine variations in workfunction or map potential across a device [15]. KPFM has been used to perform surface potential-gate bias spectroscopy [16, 17], and to resolve time dependent phenomenon like photovoltage in response to illumination [18] and the injection of charges in an oligothiophene derivative [17] and PbS nanocrystal films 19 .

Here we introduce a technique that measures both the injection and extraction of carriers in devices occurring on the millisecond to second time scale in organic field effect transistors (OFETs). By applying a sudden voltage to the back-gate of an OFET, we can watch the time-resolved screening of this applied

25 potential. The technique is sensitive to the population and depopulation of trap states that directly contribute to observed hysteresis and bias stress in OFETs, complementary to other techniques that measure trap states. We investigate a hole majority carrier, poly(3-hexylthiophene-2,5-diyl) (P3HT), and an electron majority carrier, a perylene diimide, PDI-CN2 (ActivInk N1400, from Polyera), to demonstrate the viability of this technique.

\section{Methods and Materials}

\subsection{Sample Fabrication}

Devices were fabricated in a back-gate, bottom contact configuration in order to be compatible with SPM techniques; a schematic of the device is shown in Fig- 
ure 1(a). Substrates were n-type (Sb-doped) low resistivity (0.008-0.020 $\Omega$-cm) silicon with a $200 \mathrm{~nm}$ thermally grown oxide layer (University Wafer). Source and drain electrodes were photolithographically patterned using a dual-layer of Shipley 1313 photoresist over Futurrex LOR5A. The deposited electrodes were $50 \mathrm{~nm}$ thick gold with a $3 \mathrm{~nm}$ titanium adhesion underlayer, deposited by e-

40 beam evaporation. Before depositing the organic material, the device substrates were ultrasonically cleaned in a bath sequence beginning with 3:1 deionized water:Micro-90, followed by deionized water, then acetone, and finally ethanol before a final five minute oxygen plasma clean.

For the P3HT devices, the substrates were silanized by immersion in a so45 lution of $5 \mu \mathrm{L}$ of octadecyltrichlorosilane (Acros Organics) per $\mathrm{mL}$ of toluene (anhydrous 99.9\%, Sigma Aldrich) for 1.5 hours in a PTFE dish. After immersion the substrates were rinsed in clean toluene and dried. P3HT $(96+\%$ regioregular, $>32 \mathrm{k} \mathrm{Mw}$, Rieke Metals) was added to chloroform (anhydrous, Sigma Aldrich) in a ratio of $12 \mathrm{mg} / \mathrm{mL}$ and mixed with a magnetic stirrer.

50 For PDI-CN2, the substrates were not silanized. The PDI-CN2 solution was made by adding chloroform dropwise to the vial containing the PDI-CN2 and a magnetic stirrer, until all the material dissolved at a concentration of about $5 \mathrm{mg} / \mathrm{mL}$.

The substrate was pre-wetted by dispensing chloroform (through a $0.2 \mu \mathrm{m}$ ${ }_{55}$ PTFE filter) and spinning at $3000 \mathrm{rpm}$ for $30 \mathrm{~s}$. The P3HT solution was then dispensed (through a $0.2 \mu \mathrm{m}$ filter) onto the substrate and spun at $3000 \mathrm{rpm}$ for $60 \mathrm{~s}$ (1000 rpm for PDI-CN2). The sample was then annealed at $150^{\circ} \mathrm{C}$ for 30 minutes resulting in a $30 \mathrm{~nm}$ film. Finally, the organic film from the outer 2-3 $\mathrm{mm}$ of the sample surface was mechanically removed to prevent shorting to the back-gate. In order to make a consistent electrical connection to the back gate in our measurement cell, the samples are mounted on steel chucks by mechanically removing the native oxide on the underside of the substrate with a diamond-tip scribe and placing a small amount of conductive paint (PELCO Conductive Colloidal Silver Paint) on the chuck before positioning the substrate; 65 the device is cured for at least 16 hours in vacuum before testing. This method 
of contacting the backgate results in ohmic contacts of about $30 \Omega$.

\subsection{Current-Voltage Measurements}

Characteristic current-voltage curves were obtained using a Keithley 2400 source-meter and a $6517 \mathrm{~b}$ electrometer/high resistance meter; these data were taken in a nitrogen environment before the samples were exposed to ambient conditions.

To take three-terminal measurements on the AFM, the samples were placed in a custom cell (based on the Asylum Research polymer heater stage 20]) that can be closed to protect the samples from ambient conditions (the bottom half

of the cell is shown in Figure 1(b)); nitrogen is run through the cell at a rate of $382 \mathrm{~mL} / \mathrm{min}$. Samples were exposed to air for approximately ten minutes while transferred from the glove box to the closed cell. Current-voltage measurements were taken in the closed cell using the Asylum Research AFM controller box, Kepco high voltage power supplies, and a FEMTO current-to-voltage amplifier.

\subsection{Scanning Probe Microscopy}

Topographical and KPFM measurements were performed on an Asylum Research MFP-3D AFM; Pt-coated tips were used in order to make electronic measurements (Olympus AC240TM) with a resonant frequency of $70 \mathrm{kHz}$ and a spring constant of $2 \mathrm{~N} / \mathrm{m}$. During KPFM measurements, a small amplitude AC voltage is applied to the tip, causing the tip to oscillate if the tip and sample surface are not at the same potential. To record the potential at the surface, a feedback loop applies a DC voltage $\left(\mathrm{V}_{D C}\right)$ to the tip that minimizes the oscillation; the minimal force occurs when the tip and surface are at the same potential, allowing us to record the surface potential relative to the tip. KPFM 90 is typically used to scan across an area to map the local potential difference between the tip and sample surface [15]. Fast time resolution can be achieved by positioning the tip at a single point over the organic semiconductor and recording changes in potential. We position the tip some distance from our grounded electrodes and record the potential below the tip as the back-gate is switched 
on and off in the device. In this way, we observe charges moving into and out of the film. The maximum voltage of our KPFM feedback loop is approximately $6 \mathrm{~V}$.

\section{Results and discussion}

\subsection{Control}

100

To introduce the time-resolved KPFM we start with a control sample. The geometry is identical to the finished samples but lacks the organic film. The geometry of the wafer and electrodes yields an estimated capacitance of $5 \mathrm{nF}$. The source and drain electrodes are grounded. We take a topographic scan near the electrodes and position the tip $40 \mu \mathrm{m}$ from the electrode gap edge. We then turn on the KPFM feedback to record the potential of the surface.

Figure 2 (solid black line) shows the applied gate voltage. When the AFM tip is placed over the gold electrode, there is no change in measured potential as the gate is turned on and off. In this case (blue dashed line), the electrons in the gold move too quickly for the KPFM feedback loop to register a change, and the potential remains at zero. If the tip is placed over the bare $\mathrm{SiO}_{2}$ layer (red dotted line), the measured surface potential follows the potential applied to the back gate. Charges cannot move into or through the oxide, so the unscreened gate potential is measured by the tip. By fitting an exponential to this rise in measured KPFM potential, we find a time constant of $3 \mathrm{~ms}$, which sets the time resolution of our system. We can measure charge motion in materials on the scale of milliseconds. This time resolution is set in our system by the mechanical bandwidth of the cantilever in amplitude modulation mode, corresponding to a time constant $\tau=\frac{2 Q}{f_{0}}[21]$. Typical values for our cantilevers are $f_{0} \approx 75 \mathrm{kHz}$ and $Q \approx 50$, giving a time constant $\approx 4 \mathrm{~ms}$. There is an inherent tradeoff ${ }_{120}$ in amplitude modulation mode between sensitivity and response time. Active damping, or different cantilevers, can improve response time when sensitivity is not required. 


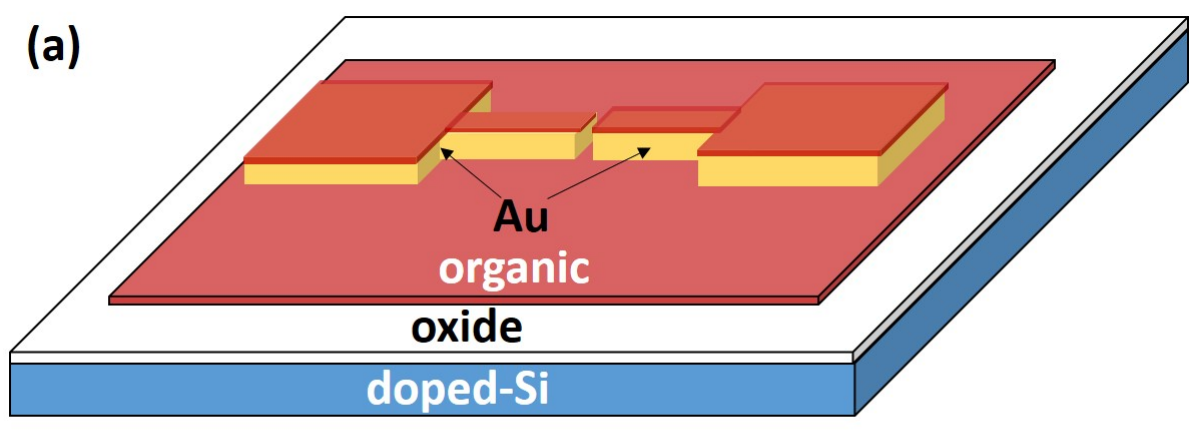

(b)

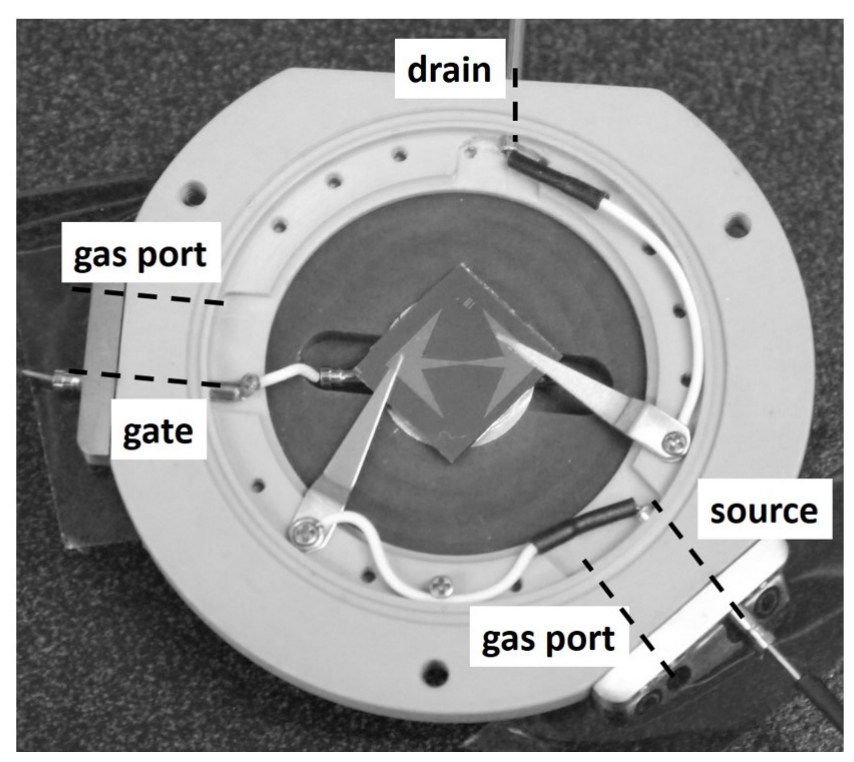

Figure 1: (a) Schematic of the FET device architecture. (b) The bottom of the closed cell. Conductive clips hold the sample in place and make the source and drain contacts; the clips are connected to wires which feed through to the outside of the cell. The gate connection is made through the steel chuck. Two additional ports permit tubes that serve as the gas inlet and outlet. 


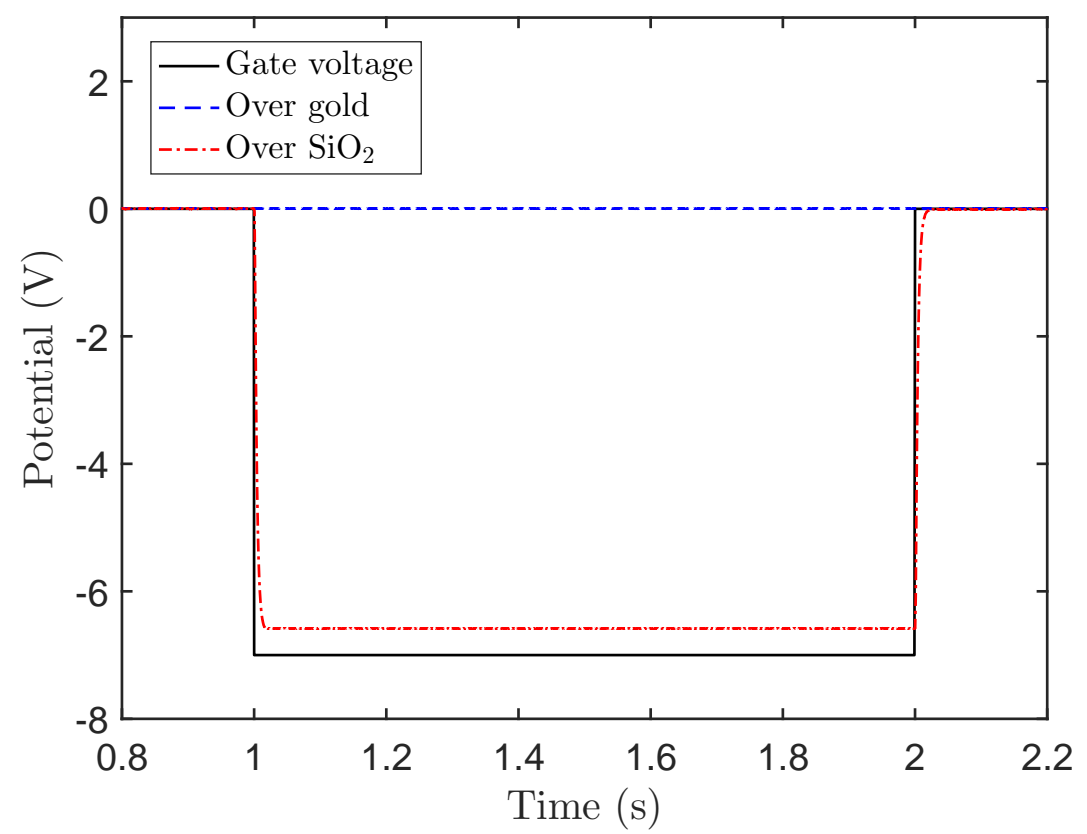

Figure 2: Characterization of the time-resolved KPFM using a control wafer with no organic film and applying a voltage to the back-gate (black solid line). When the tip is positioned above the grounded gold electrodes (blue dashed line), the measured potential is constant because the screening from the gold occurs faster than the KPFM loop can respond. When the tip is positioned over the silicon oxide surface (red dotted line), the local surface potential follows the gate voltage. The time constant describing the response time of the KPFM loop is approximately $3 \mathrm{~ms}$. 


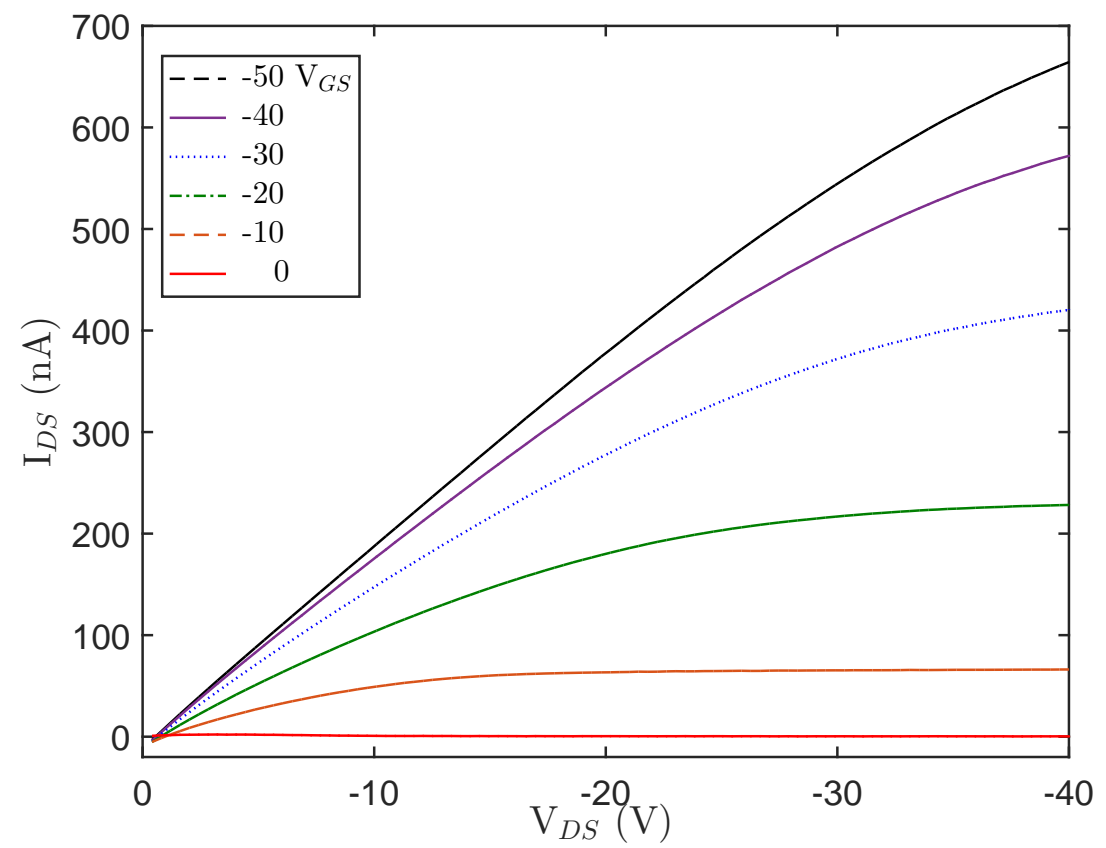

Figure 3: Typical $I_{D S}$ vs. $V_{D S}$ curves for a P3HT OFET. 


\subsection{P3HT Devices, hole majority carrier}

As shown in the output curves (Figure 3), the P3HT has p-type character;

$$
f(t)=A_{1} e^{-t / \tau_{1}}+A_{2} e^{-t / \tau_{2}}
$$


While the double exponential suggests there are two dominant physical mechanisms, meaningful quantitative physical interpretation of a four parameter fit is challenging. The weighted average time constant helps us to accurately capture the difference among the curves, given by [25]:

$$
\bar{\tau}=\frac{A_{1} \tau_{1}^{2}+A_{2} \tau_{2}^{2}}{A_{1} \tau_{1}+A_{2} \tau_{2}} .
$$

Figure 4 includes the weighted average time constants for each peak. For the negative back-gate potential, the time constant for holes entering the film $(\bar{\tau}=0.5 \mathrm{~s})$ is faster than the time constant for the holes exiting the film $(\bar{\tau}=0.6 s)$. This finding is consistent across devices regardless of age of device and air exposure, though the magnitude of the time constants may vary. The same trend is seen for the positive gate voltage. The time constant for holes exiting the film as the positive gate voltage is applied $(\bar{\tau}=2.9 \mathrm{~s})$ is longer than holes entering the film as the gate voltage returns to zero $(\bar{\tau}=0.9 s)$. We see no evidence of minority carrier transport in the current-voltage curves. The longer time constants associated with holes exiting the film could be due to either an extraction barrier (larger than any injection barrier) or to the need to empty traps in order to push the necessary number of holes out of the film. We performed spatial KPFM scans across the channel while applying a range of source-drain voltages, none of which showed any potential drops at either electrode, suggesting an ohmic contact between the Au electrodes and P3HT.

The fact that it is faster to inject holes than to extract holes can be explained by considering the prominent role that traps play in charge motion in devices like these. Given the $5 \mathrm{nF}$ capacitance of the device and the $6 \mathrm{~V}$ gate potential, approximately $10^{11}$ holes enter the film. As holes enter the film, some fall into traps of varying depths. When holes need to exit from the film, some of these holes are trapped deeply enough to impact the amount of time that it takes to move in response to the change in the gate voltage. The effect is most prominent when applying the positive gate voltage, which pushes the largest number of holes from the films, presumably emptying them from deeper traps. 


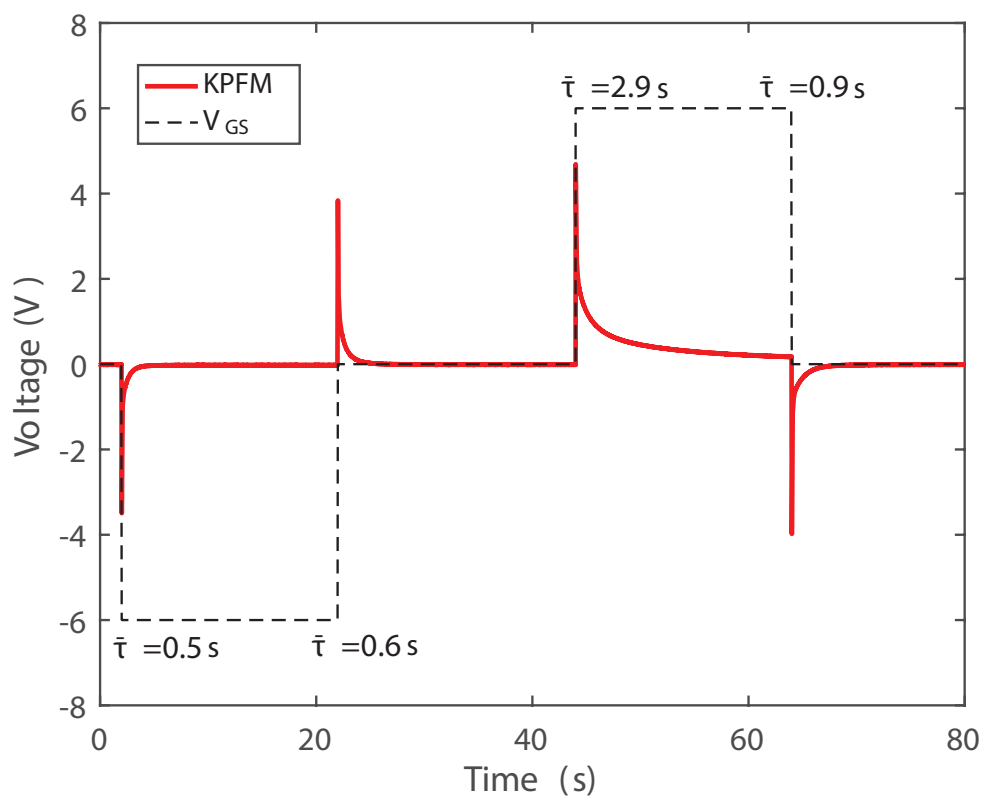

Figure 4: Time-resolved KPFM sequence for the P3HT device. The dotted black line displays the potential applied to the gate. An initial negative peak decays to a neutral potential as holes flow into the film from the electrodes. When the gate is turned off, there is initially an excess of holes (positive peak) that must leave the film. When applying a positive back-gate voltage, the second positive peak is more slowly screened as additional holes leave the film. When the positive gate voltage is removed, the initial negative potential due to too few holes is reduced as holes are quickly injected into the film. The weighted average time constant is indicated for each peak.

There is a slow migration out of the film, with a larger contribution from longlived trap states. Given the minimal current measured for $V_{G}=0 \mathrm{~V}$, there is not much mobile charge available to exit the film when a positive gate voltage is applied.

\subsection{PDI-CN2 devices, electron majority carrier}

To verify our description of the charge flow and the role of traps, we compare the p-type P3HT devices with n-type PDI-CN2 devices. We predict the same behavior for the electron majority carrier, but with longer time constants 


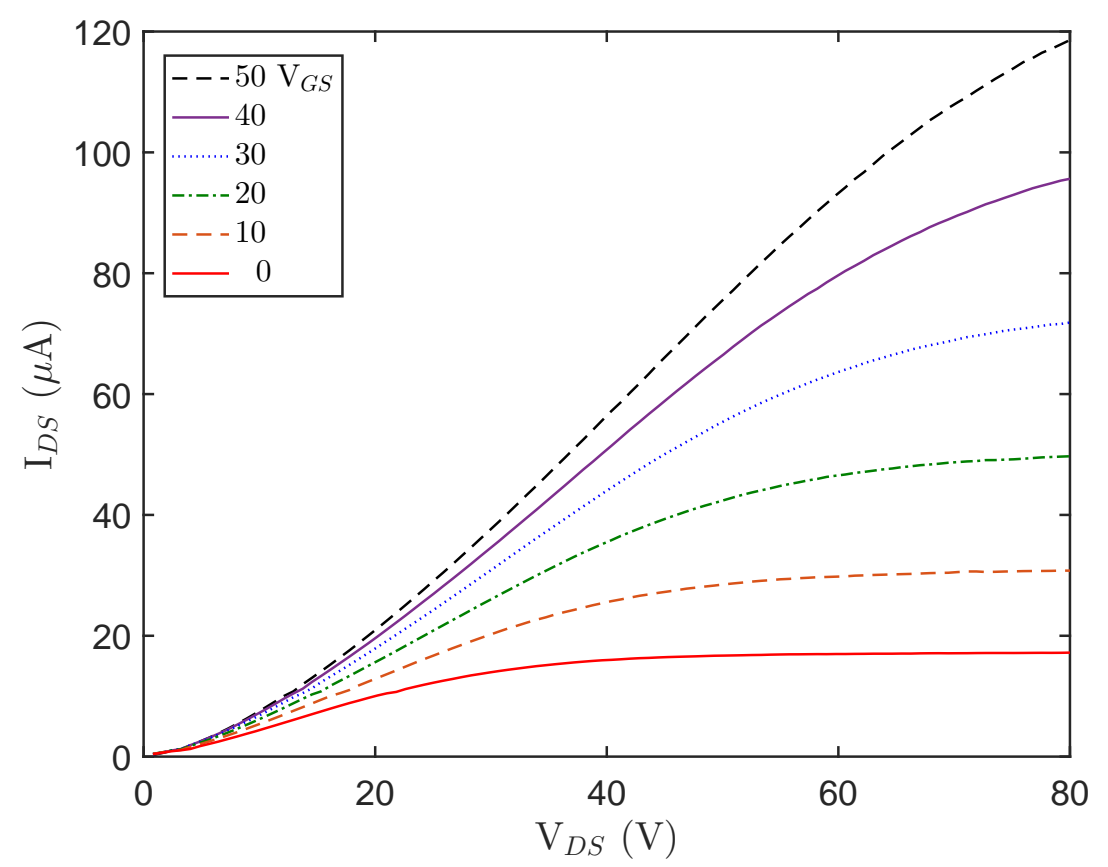

Figure 5: Typical $I_{D S}$ vs. $V_{D S}$ curves for the PDI-CN2 FETs.

associated with applying the negative gate voltage rather than the positive - it will be faster to fill traps with the majority carrier than to empty them. The characteristic output curves of the PDI-CN2 devices are shown in Figure 5 , electron mobilities were found to be $\approx 0.1 \mathrm{~cm}^{2} /(\mathrm{Vs})$, consistent with literature 26, with no evidence of hole transport; we do see evidence of a contact resistance in the IV curves. We were not able to perform KPFM scans across the channel to characterize the size or location of the resistance. Given the energy band alignments, it is more likely an electron injection barrier (workfunction of gold $-5.1 \mathrm{eV}$ and LUMO of PDI-CN2 of $-4.33 \mathrm{eV}$ ) [27, 28, which would not lead to the observed KPFM signal.

Figure 6 shows the time-resolved KPFM of the PDI-CN2 device and the weighted average time constants. As expected, it is faster to draw the majority carrier electrons into the film during the positive gate (first peak, $\bar{\tau}=0.024 \mathrm{~s}$ ) than it is to remove them (second peak, $\bar{\tau}=0.027 \mathrm{~s}$ ). The same is true for 


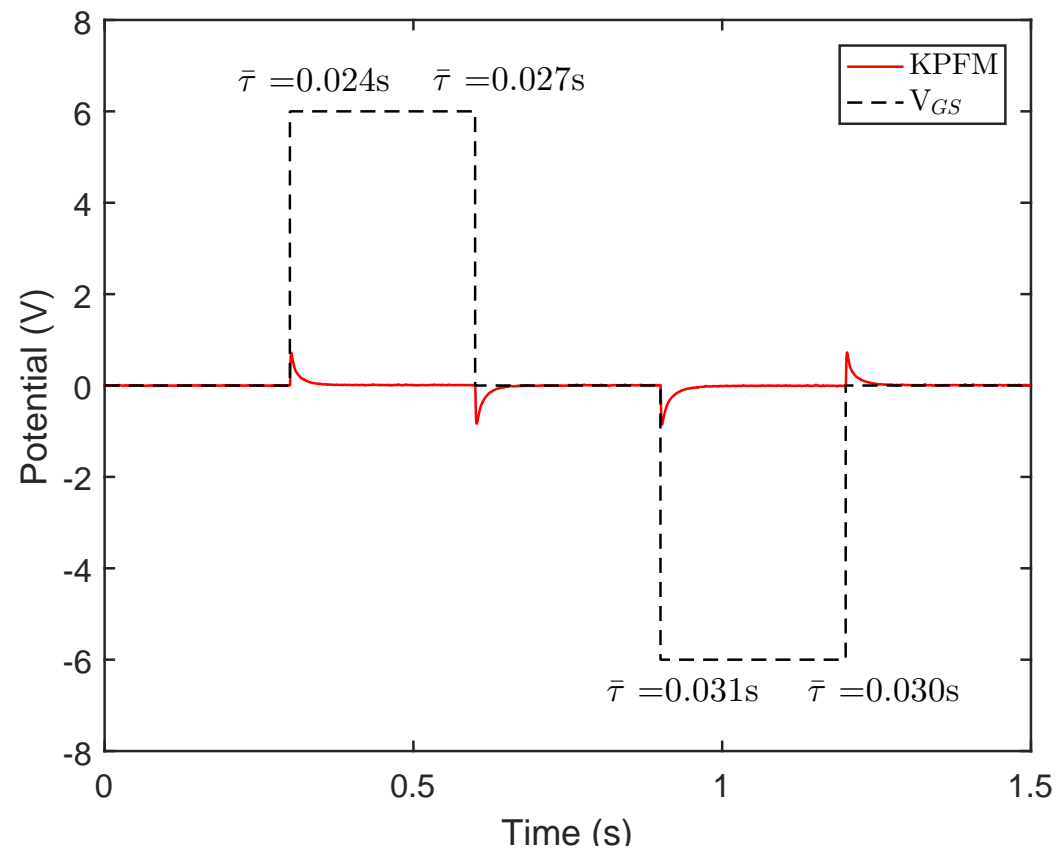

Figure 6: Time-resolved KFPM of PDI-CN2. The applied gate voltage (dotted black line) is screened by electrons injected and extracted from the film, resulting in temporary positive and negative measured surface potentials (red line) due to too many or too few electrons. Weighted time constants for each peak are indicated on the graph. 
(a)

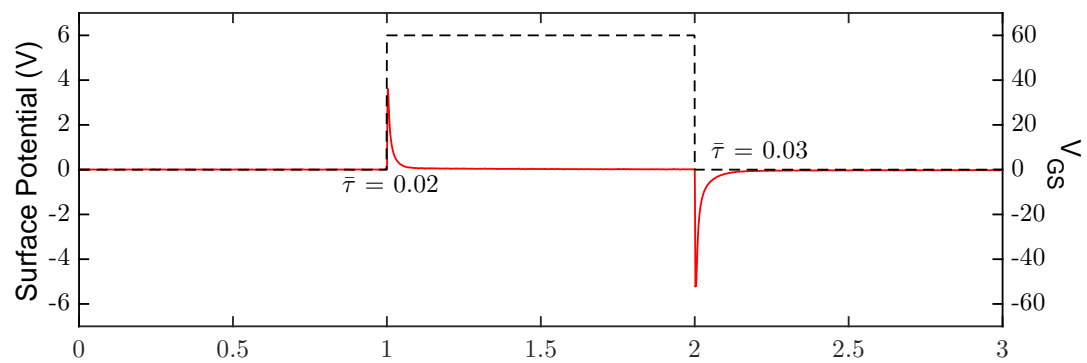

Time (s)

(b)

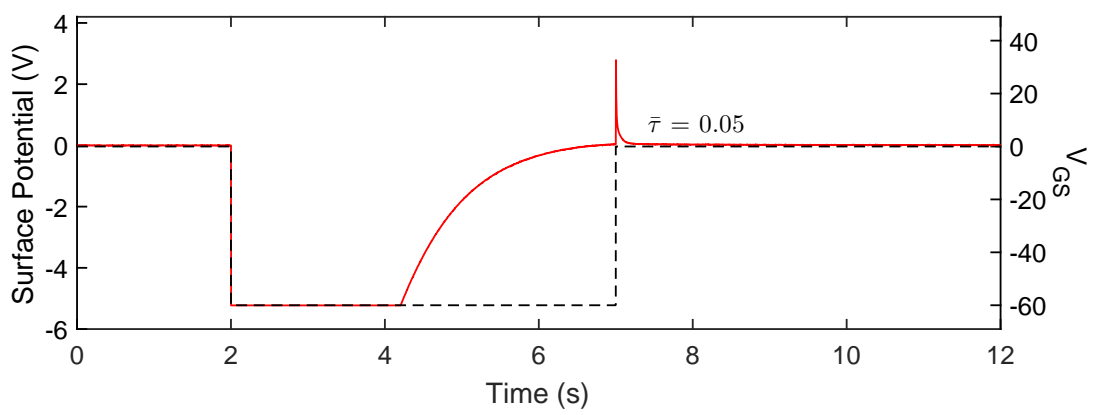

Figure 7: Time-resolved KFPM of PDI-CN2 with a larger applied gate voltage (dotted black line). (a) For $\mathrm{V}_{G S}=+60 \mathrm{~V}$, the time constants are similar to $\mathrm{V}_{G S}=+6 \mathrm{~V}$ because there are many mobile electrons in the film. (b) When $\mathrm{V}_{G S}=-60 \mathrm{~V}$, the screening of the gate voltage occurs over seconds as trapped electrons are forced to leave the film. The flat region is the maximum surface potential measurable by our KPFM feedback. As the negative voltage is removed, electrons are injected into the film much more quickly. 
applying the negative gate voltage (third peak, $\bar{\tau}=0.031 s$ ) and returning the negative gate voltage to zero (fourth peak, $\bar{\tau}=0.030 \mathrm{~s}$ ), as predicted by our interpretation of the role of trap states.

However, there are differences when comparing PDI-CN2 to the P3HT case. The peaks are much smaller in voltage, the time constants are faster, and the time constants differ by a smaller amount. The PDI-CN2 FET has a mobility that is a factor of 50 higher than P3HT, and Figure 5 shows that an electron channel exists even with zero gate voltage, indicating mobile charges are present times, since more mobile charges are available and the mobility is higher. Since drift velocity is proportional to mobility $\left(v_{d}=\mu E\right)$, in the absence of traps we would expect the time constant for screening with free carriers to be proportional to mobility, given the same electric field. The time constants of the screening $\mathrm{CN} 2$ and $0.5 \mathrm{~s}$ and $0.9 \mathrm{~s}$ for P3HT). Quoting a single mobility can be misleading, as these materials have field dependent mobilities, and traps play a role in the measured bulk mobility. We expect to see increases in time constants associated with screening when fewer mobile charges are available and trapped electrons 220 must exit the film.

To enhance the difference in time constants and the effect that the trap states play, we applied an order of magnitude larger gate voltage. Figure 7(a) shows the results with a $+60 \mathrm{~V}$ gate. There is little change in the time constants because the electrons are easily injected into the film, which already had mobile charges at zero gate voltage and mostly filled trap states. Figure 7 (b) shows a distinct contrast when applying a $-60 \mathrm{~V}$ gate. As the negative gate voltage is applied, the KPFM signal hits its maximum, preventing us from extracting a valid weighted time constant. However, the film takes about $7 \mathrm{~s}$ to fully screen the gate potential. Removing the negative gate voltage, causing electrons to rush back into the film with zero gate voltage, reveals again a short time constant on a similar scale to the positive gate voltage peaks. By applying $\mathrm{V}_{G S}=-60 \mathrm{~V}$, we deplete the film of mobile carriers, and electrons must escape longer lived traps 
to exit the film and eventually screen the gate potential.

\subsection{Bias Stress in P3HT} ments, we studied P3HT films by stepping the gate voltage in $2.5 \mathrm{~V}$ increments down to $-50 \mathrm{~V}$ and back up to zero. We repeated this measurement five times. (See Figure 8.) For each step, there is a temporary peak in the KPFM signal as holes enter or exit the film in order to return to a neutral voltage. When stepstatistically less likely to be filled and emptied are filled over time (e.g. traps 
a short physical distance into the oxide layer) 29, 24]. Repeated measurements fill these traps, reducing the number of mobile and shallowly trapped carriers. The result is that when the last of the injected holes must exit the film as the gate voltage returns to zero, the amount of time required to screen the potential from the gate increases.

\section{Conclusions}

We characterize a time-resolved KPFM technique that records the motion of charge carriers in response to a changing gate voltage, measuring the potential at the surface of the film as the carriers screen the potential of the gate. For the tip and feedback parameters used, the mechanical bandwidth primarily determines the time resolution, which is approximately $3 \mathrm{~ms}$. We demonstrate the ability to distinguish between populating and depopulating trap states, and the sensitivity of this technique to different ranges of gate voltage that push the device into different operating regimes. Repeated measurements reveal the effects of bias stress. As a scanning probe technique, KPFM offers the flexibility to determine contact resistance, and to examine location dependent variations in transport. The technique has the potential to differentiate among bulk contributions, interfaces at metal electrodes, and structures within the film such as grain boundaries. Further careful study examining a wider range of devices and the dependence on gate voltage and distance from the electrodes will more fully elucidate the contribution from the contacts, mobility, material non-uniformity, and traps.

\section{Acknowledgments}

K.A. thanks the National Science Foundation for support of this work through the NSF CAREER Award (DMR-0955348) and all authors appreciate the support received via the NSF funded Center for Hierarchical Manufacturing (CMMI1025020). 
(a)

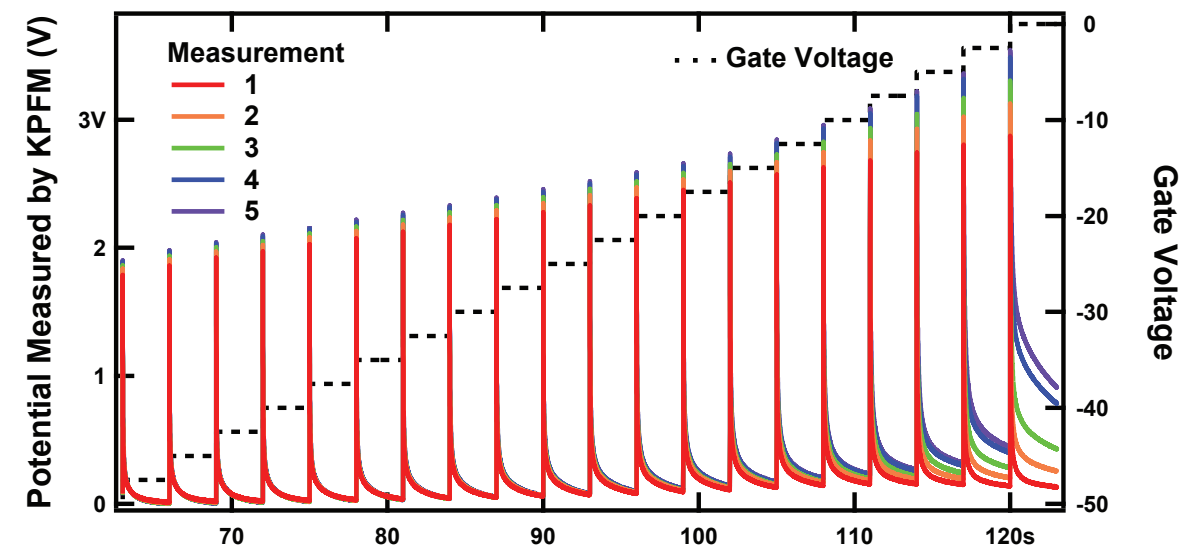

(b)

Time (s)

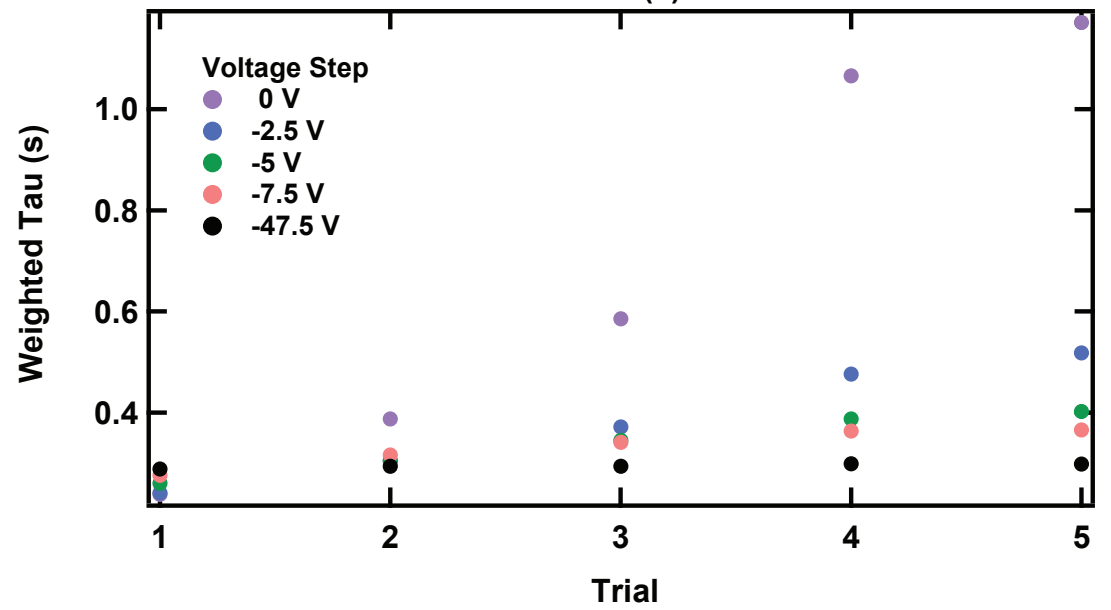

Figure 8: (a) Time-resolved KFPM of P3HT in response to stepping the gate voltage from $-50 \mathrm{~V}$ to $0 \mathrm{~V}$ in twenty steps. The measurement is repeated five times. (b) Weighted time constants for selected peaks for each of the five measurements. " 0 " indicates the step from $-2.5 \mathrm{~V}$ to $0 \mathrm{~V}$. 
The authors would like to thank Asylum Research for assisting in the customization of the code and sample stage, Andrew R. Davis for sharing his knowledge of P3HT devices to get us up and running, and Thomas Liimatainen of Mount Holyoke College for his expertise in constructing the closed cell used for this research.

\section{References}

[1] Y.-J. Lee, Y. Uk Lee, H.-W. Yeon, H.-A.-S. Shin, L. A. Evans, Y.-C.

口 Joo, Influences of semiconductor morphology on the mechanical fatigue behavior of flexible organic electronics, Applied Physics Letters 103 (24). doi:http://dx.doi.org/10.1063/1.4845995.

URL http://scitation.aip.org/content/aip/journal/apl/103/24/ $10.1063 / 1.4845995$

[2] P. J. Diemer, Z. A. Lamport, Y. Mei, J. W. Ward, K. P. Goetz, W. Li, M. M. Payne, M. Guthold, J. E. Anthony, O. D. Jurchescu, Quantitative

a analysis of the density of trap states at the semiconductor-dielectric interface in organic field-effect transistors, Applied Physics Letters 107 (10). doi:http://dx.doi.org/10.1063/1.4930310. URL http://scitation.aip.org/content/aip/journal/apl/107/10/ $10.1063 / 1.4930310$

[3] Y. Liu, J. Tolentino, M. Gibbs, R. Ihly, C. L. Perkins, Y. Liu, N. Craw$310 \quad \square$ ford, J. C. Hemminger, M. Law, PbSe quantum dot field-effect transistors with air-stable electron mobilities above $7 \mathrm{~cm}^{2} \mathrm{v}^{-1} \mathrm{~s}^{-1}$, Nano Letters 13 (4) (2013) 1578-1587, pMID: 23452235. arXiv:http://dx.doi.org/ 10.1021/n1304753n doi:10.1021/nl304753n.

URL http://dx.doi.org/10.1021/nl304753n

[4] G. H. Carey, A. L. Abdelhady, Z. Ning, S. M. Thon, O. M. Bakr, E. H. Sargent, Colloidal quantum dot solar cells, Chemical Reviews 115 (23) (2015) 12732-12763, pMID: 26106908. arXiv:http://dx.doi.org/10. 
1021/acs.chemrev.5b00063, doi:10.1021/acs.chemrev.5b00063

URL http://dx.doi.org/10.1021/acs . chemrev .5b00063

[9] Y. Wang, H.-Y. Wang, M. Yu, L.-M. Fu, Y. Qin, J.-P. Zhang, X.-C. Ai,
Trap-limited charge recombination in intrinsic perovskite film and meso-
superstructured perovskite solar cells and the passivation effect of the hole345

[5] J. Socratous, K. K. Banger, Y. Vaynzof, A. Sadhanala, A. D. Brown, A. Sepe, U. Steiner, H. Sirringhaus, Electronic structure of low-temperature solution-processed amorphous metal oxide semiconductors for thin-film transistor applications, Advanced Functional Materials 25 (12) (2015) 1873-1885. doi:10.1002/adfm.201404375. URL http://dx.doi.org/10.1002/adfm.201404375

q [6] N. K. Elumalai, C. Vijila, R. Jose, A. Uddin, S. Ramakrishna, Metal oxide semiconducting interfacial layers for photovoltaic and photocatalytic applications, Materials for Renewable and Sustainable Energy 4 (3) (2015) 1-25. doi:10.1007/s40243-015-0054-9 URL http://dx.doi.org/10.1007/s40243-015-0054-9

[7] W. Zhu, T. Low, Y.-H. Lee, H. Wang, D. B. Farmer, J. Kong, F. Xia, P. Avouris, Electronic transport and device prospects of monolayer molybdenum disulphide grown by chemical vapour deposition, Nature Communications 5 (3087). doi:10.1038/ncomms4087.

[8] D. Shi, V. Adinolfi, R. Comin, M. Yuan, E. Alarousu, A. Buin, Y. Chen, S. Hoogland, A. Rothenberger, K. Katsiev, Y. Losovyj, X. Zhang, P. A. Dowben, O. F. Mohammed, E. H. Sargent, O. M. Bakr, Low trap-state density and long carrier diffusion in organolead trihalide perovskite single crystals, Science 347 (6221) (2015) 519522. arXiv:http://science.sciencemag.org/content/347/6221/519. full.pdf, doi:10.1126/science.aaa2725

URL http://science.sciencemag.org/content/347/6221/519 transport material on trap states, Phys. Chem. Chem. Phys. 17 (2015) 
29501-29506. doi:10.1039/C5CP04360C.

URL http://dx.doi.org/10.1039/C5CP04360C

[10] H. Hirwa, V. Wagner, New insights on traps states in organic semiconductor applying illumination-free transient current

method Organic Electronics 25 (2015) 112 - 120 doi:http:

//dx.doi.org/10.1016/j.orgel.2015.06.025. URL http://www.sciencedirect.com/science/article/pii/ S1566119915002736

[11] Y. Wang, X. Qiao, D. Yang, J. Huang, J. Chen, D. Ma,

q. L. Dong, Investigation of electron transport properties in li2co3doped bepp2 thin films, Organic Electronics 26 (2015) 86 - 91. doi:http://dx.doi.org/10.1016/j.orgel.2015.07.023

(1) URL http://www.sciencedirect.com/science/article/pii/ S1566119915300458

[12] K. Vandewal, L. Goris, I. Haeldermans, M. Nesldek, K. Haenen, P. Wagner, J. Manca, Fourier-transform photocurrent spectroscopy for a fast and highly sensitive spectral characterization of organic and hybrid solar cells, Thin Solid Films 516 (20) (2008) 7135 - 7138, proceedings on Advanced Materials and Concepts for Photovoltaics \{EMRS\} 2007 Conference, Strasbourg, France. doi:http://dx.doi.org/10.1016/j.tsf.2007.12.056.

a URL http://www.sciencedirect.com/science/article/pii/ S0040609007020378

[13] D. Bozyigit, S. Volk, O. Yarema, V. Wood, Quantification of deep traps in nanocrystal solids, their electronic properties, and their influence on device

1. behavior, Nano Letters 13 (11) (2013) 5284-5288, pMID: 24164600. arXiv: http://dx.doi.org/10.1021/nl402803h, doi:10.1021/nl402803h URL http://dx.doi.org/10.1021/nl402803h

[14] J. A. Carr, S. Chaudhary, On the identification of deeper defect levels in organic photovoltaic devices, Journal of Applied Physics 114 (2013) 064509. 
[15] S. Sadewasser, T. Glatzel, Kelvin Probe Force Microscopy: Measuring and Compensating Electrostatic Forces, Springer, 2012.

[16] K. Celebi, P. J. Jadhav, K. M. Milaninia, M. Bora, M. A. Baldo, The density of states in thin film copper phthalocyanine measured by Kelvin probe force microscopy, Applied Physics Letters 93 (8) (2008) 083308, wOS:000259011900096. doi:10.1063/1.2976634.

[17] Y. Zhang, D. Ziegler, M. Salmeron, Charge Trapping States at the SiO2Oligothiophene Monolayer Interface in Field Effect Transistors Studied by Kelvin Probe Force Microscopy, Acs Nano 7 (9) (2013) 8258-8265, wOS:000330016900089. doi:10.1021/nn403750h.

[18] R. Giridharagopal, G. E. Rayermann, G. Shao, D. T. Moore, O. G. Reid, A. F. Tillack, D. J. Masiello, D. S. Ginger, Submicrosecond time resolution atomic force microscopy for probing nanoscale dynamics, Nano Letters 12 (2) (2012) 893-898, pMID: 22248070. arXiv:http://dx.doi.org/10. 1021/nl203956q, doi:10.1021/nl203956q URL http://dx.doi.org/10.1021/nl203956q

[19] Y. Zhang, Q. Chen, A. P. Alivisatos, M. Salmeron, Dynamic charge carrier trapping in quantum dot field effect transistors, Nano Letters 15 (7) (2015)

14 4657-4663, pMID: 26099508. arXiv:http://dx.doi.org/10.1021/acs. nanolett.5b01429, doi:10.1021/acs.nanolett.5b01429

URL http://dx.doi.org/10.1021/acs.nanolett.5b01429

[20] Asylum Research, PolyHeater, last accessed 4/19/16.

a URL https://www.asylumresearch.com/Products/PolyHeater/ PolyHeater.shtml

${ }_{400}$ [21] T. R. Albrecht, P. Grutter, D. Horne, D. Rugar, Frequency modulation detection using highq cantilevers for enhanced force microscope sensitivity, Journal of Applied Physics 69 (2). 
[22] M. C. Hamilton, S. Martin, J. Kanicki, Field-effect mobility of organic polymer thin-film transistors, Chemistry of Materials 16 (2004) 4699-4704. doi:10.1021/cm049613r.

URL http://dx.doi.org/10.1021/cm049613r

[23] G. Garcia-Belmonte, A. Munar, E. M. Barea, J. Bisquert, I. Ugarte, R. Pacios, Charge carrier mobility and lifetime of organic bulk heterojunctions analyzed by impedance spectroscopy, Organic Electronics 9 (5) (2008) 847 -851. doi:http://dx.doi.org/10.1016/j.orgel.2008.06.007.

口 URL http://wwW.sciencedirect.com/science/article/pii/ S1566119908001122

[24] H. H. Choi, M. S. Kang, M. Kim, H. Kim, J. H. Cho, K. Cho, Decoupling the Bias-Stress-Induced Charge Trapping in Semiconductors and GateDielectrics of Organic Transistors Using a Double Stretched-Exponential Formula, ADVANCED FUNCTIONAL MATERIALS 23 (6) (2013) 690696. doi:\{10.1002/adfm.201201545\}.

[25] J. R. Lakowicz, Principles of fluorescence spectroscopy, 3rd Edition, Springer, 2006.

[26] B. A. Jones, M. J. Ahrens, M.-H. Yoon, A. Facchetti, T. J. Marks, M. R. Wasielewski, High-mobility air-stable n-type semiconductors with processing versatility: Dicyanoperylene-3,4:9,10-bis(dicarboximides), Angewandte Chemie International Edition 43 (46) (2004) 6363-6366. doi:10.1002/ anie.200461324. URL http://dx.doi.org/10.1002/anie.200461324

[27] J. Youn, G. R. Dholakia, H. Huang, J. W. Hennek, A. Facchetti, T. J. Marks, Influence of Thiol Self-Assembled Monolayer Processing on BottomContact Thin-Film Transistors Based on n-Type Organic Semiconductors, 1 ADVANCED FUNCTIONAL MATERIALS 22 (9) (2012) 1856-1869. doi : $\{10.1002 /$ adfm. 201102312\} 
[28] J. H. Oh, Y.-S. Sun, R. Schmidt, M. F. Toney, D. Nordlund, M. Koenemann, F. Wuerthner, Z. Bao, Interplay between Energetic and Kinetic Factors on the Ambient Stability of n-Channel Organic Transistors Based on Perylene Diimide Derivatives, CHEMISTRY OF MATERIALS 21 (22) (2009) 5508-5518. doi:\{10.1021/cm902531d\}.

[29] Y. R. Liu, R. Liao, P. T. Lai, R. H. Yao, Bias-Stress-Induced Instability of Polymer Thin-Film Transistor Based on Poly(3-Hexylthiophene), IEEE TRANSACTIONS ON DEVICE AND MATERIALS RELIABILITY 12 (1) (2012) 58-62. doi:\{10.1109/TDMR.2011.2163408\}. 